\title{
More Constructive Lower Bounds on Classical Ramsey Numbers
}

\author{
Xiaodong $\mathbf{X u}{ }^{\dagger}$ Guangxi Academy of Sciences \\ Nanning, Guangxi 530007, China \\ xxdmaths@sina.com \\ Zehui Shao $\ddagger$ \\ School of Information Science \& Technology \\ Chengdu University, Chengdu 610106, China \\ kidszh_mail@163.com \\ Stanisław P. Radziszowski \\ Department of Computer Science \\ Rochester Institute of Technology, Rochester NY, USA \\ spr@cs.rit.edu
}

September 14, 2010

\begin{abstract}
We present several new constructive lower bounds for classical Ramsey numbers. In particular, the inequality $R(k, s+1) \geq$ $R(k, s)+2 k-2$ is proved for $k \geq 5$. The general construction permits us to prove that for all integers $k$, $l$, with $k \geq 5$ and $l \geq 3$, the connectivity of any Ramsey-critical $(k, l)$-graph is at least $k$, and if $k \geq l-1 \geq 1, k \geq 3$ and $(k, l) \neq(3,2)$, then such graphs are Hamiltonian. New concrete lower bounds for Ramsey numbers are obtained, some with the help of computer algorithms, including: $R(5,17) \geq 388, R(5,19) \geq 411, R(5,20) \geq 424, R(6,8) \geq 132$, $R(6,12) \geq 263, R(7,8) \geq 217, R(7,9) \geq 241, R(7,12) \geq 417$, $R(8,17) \geq 961, R(9,10) \geq 581, R(12,12) \geq 1639$, and also one three-color case $R(8,8,8) \geq 6079$.
\end{abstract}

Keywords: Ramsey numbers, connectivity AMS subject: 05C55

\footnotetext{
${ }^{\dagger}$ Supported in part by the Basic Research Fund of Guangxi Academy of Sciences (10YJ25XX01).

${ }^{\ddagger}$ Supported by the School Fund (2010XJZ27) and Science and Technology Project of Chengdu University (10RKYB041ZF-023).
} 


\section{Introduction}

For positive integers $k_{1}, \cdots, k_{m}, m \geq 2$, the classical Ramsey number $R\left(k_{1}, \cdots, k_{m}\right)$ is the smallest integer $n$ meeting the following condition: in any edge coloring of $K_{n}$ with $m$ colors, for some $i \in\{1,2, \cdots, m\}$, there exists a subgraph $K_{k_{i}}$ of $K_{n}$ whose all edges are colored with color $i$. Although the existence of Ramsey numbers $R\left(k_{1}, \cdots, k_{m}\right)$ was proved long ago [7], it is still notoriously difficult to find their exact values in nontrivial cases, or even just to obtain good bounds.

The sets of vertices and edges of a graph $G$ and their cardinalities will be denoted by $V G, E G, n(G)$ and $e(G)$, respectively. For $X \subset$ $V G$, we will denote by $G[X]$ the subgraph induced in $G$ by $X$. Any partition (coloring) of the edges of $K_{n}$ into $m$ classes avoiding $K_{k_{i}}$ in color $i$, will be called a $\left(k_{1}, \cdots, k_{m} ; n\right)$-coloring. For two colors, $m=2$, edge colorings can be considered as graphs where the second color corresponds to nonedges. We will refer to $(k, l)$-graph as a graph without $K_{k}$ and without independent sets of order $l$. Hence, the construction of any $(k, l)$-graph on $n$ vertices, i.e. a $(k, l ; n)$ coloring, will prove that $n<R(k, l)$. Any $(k, l ; R(k, l)-1)$-graph will be called Ramsey-critical $(k, l)$-graph.

Known lower and upper bounds for various types of Ramsey numbers are gathered in the dynamic survey [6] by the third author. Many constructive lower bounds for classical cases are presented in [11] and [10], and some of them are enhanced in this paper.

After reviewing previous work in Section 2, the difference between similar Ramsey numbers is studied in Section 3, where some constructive lower bounds generalizing those in [11] and [10] are presented. In particular, we show that $R(k, s+1) \geq R(k, s)+2 k-2$ for $k \geq 5$. Next, several new lower bounds for some concrete small cases are obtained directly from the general construction and others with the help of computer algorithms. The new bound $R(8,8,8) \geq 6079$ is derived in Section 4. Finally, in Section 5 we prove that for all integers $k, l$, with $k \geq 5$ and $l \geq 3$, the connectivity of any $(k, l)$-graph is no less than $k$, and that if $k \geq l-1 \geq 1, k \geq 3$ and $(k, l) \neq(3,2)$, then such graphs are Hamiltonian. 


\section{Previous work}

The following theorem and corollary were proved by constructive methods as parts of Theorems 2 and 3 in [11].

Theorem 1 [11] Given a $(k, s)$-graph $G$ and $a(k, t)$-graph $H$, for some $k \geq 3$ and $s, t \geq 2$, if both $G$ and $H$ contain an induced subgraph isomorphic to some $K_{k-1}$-free graph $M$, then

$$
R(k, s+t-1) \geq n(G)+n(H)+n(M)+1 .
$$

Corollary 1 [11] If $2 \leq s \leq t$ and $k \geq 3$, then

$$
R(k, s+t-1) \geq R(k, s)+R(k, t)+ \begin{cases}k-3, & \text { if } s=2 \\ k-2, & \text { if } s \geq 3 .\end{cases}
$$

The first inequality of Corollary 1 for $s=2, R(k, t+1) \geq R(k, t)+$ $2 k-3$, was proved by Burr et al. in 1989 [2]. Here, we will improve it for $k \geq 5$ in Corollary 3 in the next section. The following Theorem 2 and its generalization in Corollary 2 were proved in [10].

Theorem 2 [10] If $k \geq 2, s \geq 5$, then $R(2 k-1, s) \geq 4 R(k, s-1)-3$.

Corollary 2 [10] For $k_{1} \geq 5$ and $k_{i} \geq 2$, we have

$$
R\left(k_{1}, 2 k_{2}-1, k_{3}, \cdots, k_{r}\right) \geq 4 R\left(k_{1}-1, k_{3}, \cdots, k_{r}\right)-3 .
$$

In 1980, Paul Erdős wrote in [4], page 11 (using $r$ for our $R$ ):

Faudree, Schelp, Rousseau and I needed recently a lemma stating

$$
\lim _{n \rightarrow \infty} \frac{r(n+1, n)-r(n, n)}{n}=\infty
$$

We could prove (a) without much difficulty, but could not prove that $r(n+1, n)-r(n, n)$ increases faster than any polynomial of $n$. We of course expect

$$
\lim _{n \rightarrow \infty} \frac{r(n+1, n)}{r(n, n)}=C^{\frac{1}{2}}
$$


where $C=\lim _{n \rightarrow \infty} r(n, n)^{1 / n}$. Based on work reported in this paper, the best known lower bound estimate for the difference in $(a)$ seems to be barely $\Omega(n)$. Between 2007 and 2009 we asked about it several mathematicians, including Erdős' co-workers he mentioned himself, but nobody could recall the proof, not even its existence. The conclusion we are inclined to draw is that there exists no known proof of this result, though possibly it was known to Erdős. It is thus prudent to consider $(a)$ at the moment to be only a conjecture.

\section{Building-up lower bounds}

First we present a new constructive result in Theorem 3, which while similar to Theorem 1, it gives wider applicability. In the sequel, we will also show that in several cases it leads to better lower bounds for some classical two-color Ramsey numbers.

Theorem 3 Let $k \geq 4$ and $s, t \geq 2$. Given $a(k, s)$-graph $G$ and $a$ $(k, t)$-graph $H$, let $M$ be a graph isomorphic to induced subgraphs of $G$ and $H$. If the vertex set $V M$ can be partitioned into two nonempty sets, $V M=W_{1} \cup W_{2}$, so that for every $v \in V M-W_{i}$ and $i \in\{1,2\}$ there is no $K_{k-1}$ in $M\left[W_{i} \cup\{v\}\right]$, then

$$
R(k, s+t-1) \geq n(G)+n(H)+n(M)+1 .
$$

Proof. Let $p=n(G), q=n(H), m=n(M)$, and $m_{i}=\left|W_{i}\right|, m_{i}>0$ for $i \in\{1,2\}$, so we have $m=m_{1}+m_{2}$. First, we will construct a graph $F$ on the vertex set $V G \cup V H \cup W_{1} \cup W_{2}$ and then prove that it contains no $K_{k}$ and no independent sets of order $s+t-1$. Since $F$ has the right number of vertices this will complete the proof of the theorem.

Let $U=V G=\left\{u_{1}, \cdots, u_{p}\right\}, V=V H=\left\{v_{1}, \cdots, v_{q}\right\}$ and $W=$ $V M=\left\{w_{1}, \cdots, w_{m}\right\}$. Without loss of generality, we can assume that the graphs $G, H$ and $M$ are labeled so that their parts induced on $U_{1}=\left\{u_{j} \mid 1 \leq j \leq m_{1}\right\}, U_{2}=\left\{u_{j} \mid m_{1}+1 \leq j \leq m_{1}+m_{2}\right\}$, $V_{1}=\left\{v_{j} \mid 1 \leq j \leq m_{1}\right\}, V_{2}=\left\{v_{j} \mid m_{1}+1 \leq j \leq m_{1}+m_{2}\right\}$, $W_{1}=\left\{w_{j} \mid 1 \leq j \leq m_{1}\right\}$ and $W_{2}=\left\{w_{j} \mid m_{1}+1 \leq j \leq m_{1}+m_{2}\right\}$, are isomorphic under the mapping $u_{j} \rightarrow v_{j} \rightarrow w_{j}$. 
The set of edges of $F$ contains the edges of the graphs $G, H$, $M\left[W_{1}\right]$ and $M\left[W_{2}\right]$. The other edges in $E F$ are defined as follows. For $i \in\{1, \cdots, m\}, j \in\{1, \cdots, p\}, i \neq j$, if $\left(u_{i}, u_{j}\right) \in E G$ then $\left(w_{i}, u_{j}\right) \in E F$, and for $i \in\{1, \cdots, m\}, j \in\{1, \cdots, q\}, i \neq j$, if $\left(v_{i}, v_{j}\right) \in E H$ then $\left(w_{i}, v_{j}\right) \in E F$. Finally, $E F$ contains the edges of a matching $\left(u_{i}, v_{i}\right)_{i=1, \cdots, m}$ between $U_{1} \cup U_{2}$ and $V_{1} \cup V_{2}$. Note that $E F$ doesn't contain any edges between $W_{1}$ and $W_{2}$.

Suppose that there is a complete subgraph $K_{k}$ in $F$ induced by a set $S \subset V F$. Consider the parts of $S$ and its sizes, $S_{i} \subset S, s_{i}=\left|S_{i}\right|$ for $1 \leq i \leq 4$, as follows:

$$
\begin{aligned}
& S_{1}=S \cap V G, \quad S_{2}=S \cap V H, \\
& S_{3}=S \cap W_{1}, \quad S_{4}=S \cap W_{2},
\end{aligned}
$$

Case $1\left(s_{1}, s_{2}>0\right)$. Observe that we must have $s_{1}=s_{2}=1$, since the only edges in $E F$ between $V G$ and $V H$ form a partial matching. Hence we have that $u_{j} \in S_{1}$ and $v_{j} \in S_{2}$ for some special $1 \leq j \leq m$. Further, since $s_{3} * s_{4}=0$ we can assume that $s_{3}=k-2, S_{4}=\emptyset$. This in turn would imply that the induced graph $M\left[S_{3} \cup\left\{w_{j}\right\}\right]$ contains $K_{k-1}$, contrary to the assumptions od the theorem.

Case $2\left(s_{1} * s_{2}=0\right)$. Without loss of generality assume that $s_{2}=$ $s_{4}=0$. From the construction we can easily see that the induced subgraph $G\left[S_{1} \cup\left\{u_{j} \in V G \mid w_{j} \in W_{1}\right\}\right]$ contains $K_{k}$, which is a contradiction.

It remains to be shown that $F$ has no independent sets of order $s+t-1$. Here, we will use a method very similar to the proof of Theorem 2 (Theorem 9 in [10]). Consider any independent set $I$ in $F$, and its parts $I_{i}$, as follows:

$$
\begin{aligned}
& I_{1}=I \cap V G, \quad I_{2}=I \cap V H, \\
& I_{3}=I \cap W_{1}, \quad I_{4}=I \cap W_{2}, \\
& I_{5}=\left\{u_{j} \in I_{1} \mid w_{j} \in I_{3}\right\} \cup\left\{v_{j} \in I_{2} \mid w_{j} \in I_{3}\right\}, \\
& I_{6}=\left\{u_{j} \in I_{1} \mid w_{j} \in I_{4}\right\} \cup\left\{v_{j} \in I_{2} \mid w_{j} \in I_{4}\right\}, \\
& I_{7}=I_{1} \backslash\left(I_{5} \cup I_{6}\right), \quad I_{8}=I_{2} \backslash\left(I_{5} \cup I_{6}\right) .
\end{aligned}
$$

Directly from the construction it can be concluded that

$$
\left|I_{3} \cup I_{6} \cup I_{7}\right| \leq s-1 \text { and }\left|I_{4} \cup I_{5} \cup I_{8}\right| \leq t-1 .
$$


by considering isomorphic embedding of all parts $I_{j}$ into the vertex set $V G$ and $V H$, respectively. Since clearly $I=\bigcup_{i=1}^{4} I_{i}=\bigcup_{i=3}^{8} I_{i}$, we obtain $|I| \leq s+t-2$. This finishes the proof of the theorem.

For nontrivial choices of the graphs $G, H$ and $M$ the construction will produce triangles, so we need $k \geq 4$. Even in the case of $k=4$, when $M$ is $K_{3}$-free, one should use simpler construction of Theorem 1. In the computational results presented in the sequel we have also used a slightly weaker version of Theorem 3 . Namely, it is sufficient to find any partition of the vertex set $V M$ into $W_{1} \cup W_{2}$, so that neither of $M\left[W_{1}\right]$ or $M\left[W_{2}\right]$ contains a $K_{k-2}$. Then the assumptions of Theorem 3 hold, and thus the same construction works. Another observation related to this theorem concerns a possible special case, namely $H=M=K_{k-1}$, and the corresponding partition of $V M$ into $K_{2}$ and $K_{k-3}$. If we use $t=2$ and any critical $(k, s)$-graph as $G$, then for $k \geq 5$ we obtain a $(k, s+1)$-graph $F$ (note that for $k=4$ this special case of the partition doesn't satisfy the assumptions). This implies $R(k, s+1) \geq R(k, s)+2 k-2$, which improves by one Corollary 1 for $s=2$, as in the second part of the next corollary.

Corollary 3 If $k \geq 5$ and $2 \leq s, t$, then $R(k, s+t-1) \geq R(k, s)+$ $R(k, t)+k-2$. In particular, we have $R(k, s+1) \geq R(k, s)+2 k-2$.

We are ready to derive some new lower bounds for two-color Ramsey numbers, as in the following theorem. The new bounds improve on those given in [6].

Theorem $4 R(6,12) \geq 263, R(7,8) \geq 217, R(7,12) \geq 417$, $R(9,10) \geq 581$, and $R(12,12) \geq 1639$. 
Proof. Using Corollary 3 with the best known lower bounds for $R(k, s)$ recorded in [6], we obtain:

$$
\begin{array}{rll}
R(6,12) & \geq R(6,11)+2 \times 6-2 & \geq 263, \\
R(7,8) & \geq R(7,7)+2 \times 7-2 & \geq 217, \\
R(7,12) & \geq R(7,11)+2 \times 7-2 & \geq 417, \\
R(9,10) & \geq R(9,9)+2 \times 9-2 & \geq 581, \\
R(11,12) & \geq R(11,11)+2 \times 11-2 \geq 1617, \\
R(12,12) & \geq R(12,11)+2 \times 12-2 \geq 1639 .
\end{array}
$$

The next improvements of the lower bounds required some help of computer algorithms. The results are collected in Theorem 5. All of them improve over the bounds from [8] or listed in [6].

Theorem $5 R(5,17) \geq 388, R(5,19) \geq 411, R(5,20) \geq 424$, $R(6,8) \geq 132, R(7,9) \geq 241$, and $R(8,17) \geq 961$.

Proof. Let us define the graph $G_{387}$ by taking as its edges just one color in the tripling construction with $p=127$, which builds up on the well known Mathon's construction [5], as described in [9]. With some computations, it can be checked that $G_{387}$ is a $(5,17 ; 387)$ graph establishing the bound $R(5,17) \geq 388$, which improves over the previously listed value of 385 [6]. In the following, we will use the same graph in two other constructions.

\begin{tabular}{rrrrrrr}
\hline$k$ & $s$ & $t$ & $|V G|$ & $|V H|$ & $|V M|$ & $|V F|$ \\
\hline 6 & 6 & 3 & 101 & 17 & 13 & 131 \\
7 & 7 & 3 & 204 & 22 & 14 & 240 \\
5 & 17 & 3 & 387 & 13 & 10 & 410 \\
5 & 17 & 4 & 387 & 24 & 12 & 423 \\
\hline
\end{tabular}

Table 1. Parameters of Theorem 3 used in Theorem 5.

Each of the rows in Table 1 presents sizes of the parameters used in an application of Theorem 3, for which a proper partition 
of the set $V M$ was found. The graph $G_{387}$ was used as $G$ in the third and fourth row. The other cases of $G$ and all graphs $H$ were taken from the standard set of known largest Ramsey graphs for the corresponding parameters (cf. $[6,11]$ ). The common subgraph $M$ and the partition $V M=W_{1} \cup W_{2}$ were found in each case with the help of computer heuristics (larger common parts possibly could be found). Four lower bounds in Theorem 5 are one larger than the entries in the last column of Table 1 . Finally, we obtain $R(8,17) \geq$ 961 from $R(7,9) \geq 241$ by applying Theorem 2 .

\section{New lower bound for $R(8,8,8)$}

Theorem 6 below is a special case of a more general result in [10]. The Paley graph $Q_{p}$ is defined for primes $p$ of the form $4 t+1$. The vertex set of $Q_{p}$ is $\mathcal{Z}_{p}$, and the vertices $x$ and $y$ are joined by an edge if and only if $x-y$ is a square modulo $p$.

Theorem 6 [10] For a prime $p$ of the form $4 t+1$, let $\alpha_{p}$ be the order of the largest clique in the Paley graph $Q_{p}$. Then

$$
R\left(3, \alpha_{p}+2, \alpha_{p}+2\right) \geq 6 p+3 .
$$

Following the construction used in the proof of Theorem 2 in [10], one can easily conclude the following lemma by using Corollary 2 .

Lemma 1 Suppose $k \geq 2, s, t \geq 4$. If $G$ is a $(k, s, t ; n)$-coloring, in which the subgraph induced by the edges in color 1 is $d$-regular, then there exists a $(2 k-1, s, t+1 ; 4 n)$-coloring, in which the subgraph induced by the edges in color 1 is $(n+3 d+1)$-regular.

The new lower bound on $R(8,8,8)$ will be obtained using Theorem 6 and Lemma 1 above.

Theorem $7 \quad R(8,8,8) \geq 6079$. 
Proof. Using Theorem 6 with $p=101$ we can obtain a $(3,7,7 ; 608)$ coloring, furthermore, by following the construction of the proof in [10], we can see that it is regular of degree 202 in color 1 . Next, by Lemma 1 we can build a $(5,7,8 ; 2432)$-coloring, which is regular of degree $1215=608+3 \times 202+1$. Using Lemma 1 once more, we make a $(9,8,8 ; 9728)$-coloring, which is regular of degree 6078 in color 1 . Now the neighborhood in color 1 of any vertex in the last coloring induces a $(8,8,8 ; 6078)$-coloring, which completes the proof.

\section{Connectivity of Ramsey graphs}

Beveridge and Pikhurko in [1], using Theorem 1, proved that for any $k, l \geq 3$, the connectivity of any Ramsey-critical $(k, l)$-graph is no less than $k-1$. Here, in Theorem 8 , for $k \geq 5$, we increase this bound on connectivity to $k$. Similarly, the following Corollary 4 improves over a corollary in [1] on Ramsey-critical graphs which are Hamiltonian. Our results directly depend on Corollary 3, in a way that they could be further strengthened if the lower bound of Corollary 3 is improved.

Theorem 8 If $k \geq 5$ and $l \geq 3$, then the connectivity of any Ramsey-critical $(k, l)$-graph is no less than $k$.

Proof. Suppose some graph $F$ is a Ramsey-critical $(k, l)$-graph, and its connectivity $\kappa$ is less than $k$, for some $k \geq 5$ and $l \geq 3$. Thus, by the result of Beveridge and Pikhurko, $\kappa=k-1$. Let $C$ be the cut-set of $V F,|C|=k-1$, such that $G[V F \backslash C]$ is disconnected. Let $V_{1} \cup V_{2} \cup C$ be the corresponding partition of $V F$. Consider the graphs $G_{1}=F\left[V_{1}\right], n_{1}=\left|V_{1}\right|$ and $G_{2}=F\left[V_{2}\right], n_{2}=\left|V_{2}\right|$, and let $s-1$ and $t-1$ be their independence numbers, respectively. Clearly, we have that $G_{1}$ is a $\left(k, s ; n_{1}\right)$-graph, $G_{2}$ is a $\left(k, t ; n_{2}\right)$-graph, $n_{1}+n_{2}+(k-1)=|V F|=R(k, l)-1$, and $(s-1)+(t-1)<l$. This implies

$$
R(k, s)+R(k, t)-2 \geq n_{1}+n_{2}=R(k, l)-k .
$$

Thus by Corollary 3 and monotonicity of Ramsey numbers we have $s+t-1=l$. Furthermore, now it also easily follows that $n_{1}=$ 
$R(k, s)-1$ and $n_{2}=R(k, t)-1$, i.e. both $G_{1}$ and $G_{2}$ are Ramseycritical for $(k, s)$ and $(k, t)$, respectively.

Observe that for any vertex $v \in C$, the induced subgraph $F\left[V_{1} \cup\{v\}\right]$ must have an independent set of $s$ vertices containing $v$, since $G_{1}$ is Ramsey-critical for $(k, s)$. Similarly, $F\left[V_{2} \cup\{v\}\right]$ has an independent set of $t$ vertices containing the same $v$. Thus, since $C$ is a cut-set, we have found an independent set of $s+t-1=l$ vertices in graph $F$, which contradicts the assumption that $F$ is a $(k, l)$-graph. Therefore $\kappa \geq k$.

Corollary 4 If $k \geq l-1 \geq 1$ and $k \geq 3$, except $(k, l)=(3,2)$, then any Ramsey-critical $(k, l)$-graph is Hamiltonian.

Proof. For $l=2$ the only Ramsey-critical $(k, l)$ graphs are complete $K_{k-1}$, which are Hamiltonian for $k \geq 4$. For $k \geq 5$ and $l \geq 3$, by Theorem 8 , any Ramsey-critical $(k, l)$-graph $F$ has connectivity $\kappa \geq k$. Theorem 1 in [3], by Chvátal and Erdős, states that any $k$-connected graph on at least three vertices, without independent sets of order $k+1$, is Hamiltonian. Thus, for $k \geq l-1$, a direct application of the latter implies that $F$ has a Hamiltonian circuit.

The remaining cases of $(k, l)$ are $(3,4),(3,3),(4,3),(4,4)$ and $(4,5)$ for the Ramsey-critical graphs of orders 8, 5, 8, 17 and 24, respectively. All $(3,4 ; 8)-,(3,3 ; 5)-,(4,3 ; 8)$ - and $(4,4 ; 17)$-graphs are known (cf. [6]). There are 3, 1, 3 and 1 of them, respectively, and they can be easily checked to be Hamiltonian. Let $F$ be any $(4,5 ; 24)$ graph. We will prove that it has connectivity $\kappa \geq 5$ (bound 4 would suffice), so again Theorem 1 in [3] will imply that $F$ is Hamiltonian. Suppose that the graph $F$ has a cut-set $C$ of 4 vertices disconnecting $F$, and let $V_{1} \cup V_{2} \cup C$ be the corresponding partition of the vertex set $V F$ (using the same notation as in the proof of Theorem 8). Since $R(4,2)=4, R(4,3)=9$ and $R(4,4)=18$, we can easily see that the only possible parameters of this partition are $n_{1}=3, s=2, n_{2}=17$, $t=4$ and $G_{1}=K_{3} . \quad R(4,4)=18$ implies that $F$ has minimum degree at least 6 , and thus each vertex in $V_{1}$ must be connected to all 4 vertices in $C$. Hence each vertex in $C$ forms a $K_{4}$ together with $V_{1}$, which is a contradiction. 
In particular, for $k \geq 3$, all diagonal Ramsey-critical $(k, k)$-graphs are Hamiltonian. It is an interesting open question for which $k<l-1$ Ramsey-critical $(k, l)$-graphs remain Hamiltonian. We expect it to be true at least when $k$ is sufficiently close to $l$.

\section{References}

[1] Beveridge A. and Pikhurko O., On the Connectivity of Extremal Ramsey Graphs. Australasian Journal of Combinatorics, 41 (2008), 57-62.

[2] Burr S. A., Erdős P., Faudree R. J. and Schelp R. H., On the Difference Between Consecutive Ramsey Numbers. Utilitas Mathematica, 35 (1989), 115-118.

[3] Chvátal V. and Erdős P., A Note on Hamiltonian Circuits. Discrete Mathematics, 2 (1972), 111-113.

[4] Erdős P., Some New Problems and Results in Graph Theory and Other Branches of Combinatorial Mathematics. Combinatorics and Graph Theory (Calcutta 1980), Berlin-NY Springer, LNM 885 (1981), 9-17.

[5] Mathon R., Lower Bounds for Ramsey Numbers and Association Scheme. Journal of Combinatorial Theory, Series B, 42 (1987), 122-127.

[6] Radziszowski S. P., Small Ramsey Numbers. Electronic Journal of Combinatorics, Dynamic Survey 1, revision $\sharp 12$, August 2009, http://www. combinatorics.org.

[7] Ramsey F. P., On a Problem of Formal Logic. Proceedings of the London Mathematical Society, 30 (1930), 264-286.

[8] Shao Zehui, Xu Jin and Pan Linqiang, Lower Bounds on Ramsey Numbers $R(6,8), R(7,9)$ and $R(8,17)$. Ars Combinatoria, 94 (2010), 55-59. 
[9] Xu Xiaodong and Radziszowski S. P., An Improvement to Mathon's Cyclotomic Ramsey Colorings. Electronic Journal of Combinatorics, $\mathrm{N} \sharp 1, \quad 16(1)$ (2009), 5 pages, http://www . combinatorics.org.

[10] Xu Xiaodong, Xie Zheng, Exoo G. and Radziszowski S. P., Constructive Lower Bounds on Classical Multicolor Ramsey Numbers, Electronic Journal of Combinatorics, R $\sharp 35,11$ (2004), 24 pages, http://www.combinatorics.org.

[11] Xu Xiaodong, Xie Zheng and Radziszowski S. P., A Constructive Approach for the Lower Bounds on the Ramsey Numbers $R(s, t)$, Journal of Graph Theory, 47 (2004), 231-239. 